DRAFT VERSION NOVEMBER 10, 2018

Preprint typeset using $\mathrm{LAT}_{\mathrm{E}} \mathrm{X}$ style emulateapj

\title{
COMPLETENESS IN PHOTOMETRIC AND SPECTROSCOPIC SEARCHES FOR CLUSTERS
}

\author{
Martin White ${ }^{1}$ and C.S. KochaneK ${ }^{2}$ \\ ${ }^{1}$ Departments of Physics and Astronomy, University of California, Berkeley, CA 94720 \\ ${ }^{2}$ Harvard-Smithsonian Center for Astrophysics, 60 Garden St., Cambridge, MA 02138 \\ email: mwhite@astron.berkeley.edu, ckochanek@cfa.harvard.edu \\ Draft version November 10, 2018
}

\begin{abstract}
We investigate, using simulated galaxy catalogues, the completeness of searches for massive clusters of galaxies in redshift surveys or imaging surveys with photometric redshift estimates, i.e. what fraction of clusters $\left(M>10^{14} h^{-1} M_{\odot}\right)$ are found in such surveys. We demonstrate that the matched filter method provides an efficient and reliable means of identifying massive clusters even when the redshift estimates are crude. In true redshift surveys the method works extremely well. We demonstrate that it is possible to construct catalogues with high completeness, low contamination and both varying little with redshift.
\end{abstract}

Subject headings: cosmology: theory - large-scale structure of Universe

\section{INTRODUCTION}

Clusters of galaxies are one of our most important cosmological probes. As the most recent objects to form in the universe their number density and properties are exquisitely sensitive to our modeling assumptions. Their composition accurately reflects the mix of matter in the universe. They are bright and can be "easily" seen to large distances, allowing constraints on the crucial interval $0<z \lesssim 1$ where the universal expansion changes from deceleration to acceleration. They are located close to their formation site. Being bright and sparse they are excellent tracers of the large-scale structure - they are highly biased so their clustering is easy to measure and is much more straightforwardly computed from theory than that of galaxies.

However, constructing large samples of massive clusters for statistical analyses remains a difficult task. The original samples (e.g. Abell 1958; Dalton et al. 1992; Lumsden et al. 1996; White et al. 1999) were selected on the basis of projected galaxy overdensity, but it was quickly realized that such surveys suffer from projection effects and the large scatter between optical richness and cluster mass (for recent theoretical studies see e.g. van Haarlem, Frenk \& White 1997; Reblinsky \& Bartelmann 1999). For this reason attention has broadened to include searches in complete redshift surveys (e.g. Huchra \& Geller 1982; Geller \& Huchra 1983; Ramella et al. 1994; Ramella, Pisani \& Geller 1997), surveys at X-ray wavelengths (Gioia et al. 1990; Edge et al. 1990: Henry \& Arnaud 1991; Rosati et al.1995: Jones et al. 1998, Ebeling et al. 1998; Vikhlinin et al. 1998; Romer et al.2000; Henry 2000; Blanchard et al. 2000; Scharf et al. 2000), and using the SunyaevZel'dovich effect (Carlstrom et al. 2000). More recently there has been significant progress in optical surveys however, both in terms of data quality and algorithmic sophistication. The introduction of accurate, multi-color photometry has allowed estimation of "photometric redshifts" which can mitigate many of the problems of foregroundbackground contamination and carefully applied filters can find cluster signals with even low numbers of cluster galaxies.

In this work we report preliminary investigations into how well a deep, multi-color optical survey would find the most massive clusters of galaxies. We envision this as a first step in a programme which would then obtain multiwavelength information about a sample so selected in order to constrain the evolution of the mass function. We contrast this with the yield expected from a shallower redshift survey such as could be done with the Hectospec instrument on the MMT (see Geller 1994).

\section{CLUSTERS AND DARK ENERGY}

A recent motivation for revisiting this question, and for investigating strategies which can allow us to construct a large, well characterized sample of the rarest clusters over the widest area possible, is the ability of clusters to shed light on the nature of the dark energy believed to be causing the accelerated expansion of the universe. The nature of this dark energy is one of the most vexing problems in cosmology and one with strong implications for our understanding of fundamental physics.

Since the dark energy is predicted to be smooth, except possibly near the horizon scale, all of its cosmological effects come in through its effect on the expansion rate $H(z)$. Specifically it alters the distance-redshift relations, cosmological volumes and the growth of perturbations, all of which are integrals of the inverse Hubble parameter over redshift. In order to best constrain the dark energy it is desirable to probe the crucial redshift range $z \simeq 0-1$, where it begins to noticeably affect the expansion rate, with as much resolution in redshift as possible. Several authors (most recently Haiman, Mohr \& Holder 2001) have suggested using the counts of clusters of galaxies to probe the evolution of the dark energy in this redshift range.

The strongest cosmological constraints come from relatively massive clusters, which are intrinsically rare. In order to construct a large sample of massive clusters at lower redshifts $(z \lesssim 1$; where detailed followup observations are conceivable), we need to cover a large area of sky. This is difficult to do with existing facilities for X-ray or Sunyaev-Zel'dovich (SZ) observations. Such a sample, selected optically, would provide a much needed complement to the higher redshift clusters found by SZ surveys over smaller areas of the sky. Once plausible cluster candidates have been found, multi-wavelength followup is possible (and necessary) to help pin down the 'local' sample 
and the normalization of the scaling relations which can convert observables into cluster mass.

\section{SIMULATED OBSERVATIONS}

A realistic search for clusters requires a good match to the spatial distribution of galaxies and to their mean density, rather than a thorough understanding of galaxy formation. We use high resolution N-body simulations for the evolution of the dark matter, described in $\$ 3.1$, to provide the large scale structure and clustering of the matter distribution. We find that N-body based models are significantly better than Poisson models in describing the fluctuations in the galaxy background which are important in cluster finding. Next, we populated the dark matter halos with galaxies as described in $\$ 3.2$. Finally, we produce a simulated observational catalog as described in $\$ 3.3$. Some of the limitations of our procedure are discussed in $\$ 3.4$.

\subsection{N-body simulation}

On large scales (Mpc and above) the distribution of galaxies will trace that of the dark matter, so we can use N-body simulations to provide a model for the large scale structure and the initial formation of gravitationally bound halos. We have run a $256^{3}$ particle simulation of a $\Lambda \mathrm{CDM}$ model in a $200 h^{-1} \mathrm{Mpc}$ box using the TreePM-SPH code (White et al. 2001) operating in collisionless (dark matter only) mode. This simulation represents a large cosmological volume, to include a fair sample of rich clusters, while maintaining enough mass resolution to identify galactic mass halos (see $\$ 3.2$ ). Because it provides a reasonable fit to a wide range of observations, including the present day abundance of rich clusters of galaxies (Pierpaoli, Scott \& White 2001), we have simulated the "concordance cosmology" of Ostriker \& Steinhardt (1995), which has $\Omega_{\mathrm{m}}=0.3, \Omega_{\Lambda}=0.7, H_{0}=100 h \mathrm{kms}^{-1} \mathrm{Mpc}^{-1}$ with $h=0.67, \Omega_{\mathrm{B}}=0.04, n=1$ and $\sigma_{8}=0.9$ (corresponding to $\left.\delta_{H}=5.02 \times 10^{-5}\right)$. The simulation was started at $z=50$ and evolved to the present with the full phase space distribution dumped every $100 h^{-1} \mathrm{Mpc}$ from $z \simeq 2$ to $z=0$. The gravitational force softening was of a spline form (e.g. Hernquist \& Katz 1989), with a "Plummer-equivalent" softening length of $28 h^{-1} \mathrm{kpc}$ comoving. The particle mass is $4 \times 10^{10} h^{-1} M_{\odot}$ allowing us to find bound halos with masses several times $10^{11} h^{-1} M_{\odot}$ and giving many, many particles in a cluster mass halo $\left(>10^{14} h^{-1} M_{\odot}\right)$ to begin to resolve substructure.

We identify the real clusters in the sample using the $3 \mathrm{D}$ dark matter distribution and the friends-of-friends (FoF) algorithm. For each cluster we calculate directly from the $3 \mathrm{D}$ distribution the mass (we use $M_{200}$, the mass enclosed within a radius, $r_{200}$, within which the mean density is 200 times the critical density at that redshift), velocity dispersion etc. so we can understand our selection in terms of the intrinsic, rather than projected, cluster properties. We define the center of a cluster as the position of the potential minimum, calculating the potential using only the particles in the FoF group. This proved to be more robust than using the center of mass, as the potential minimum coincided closely with the density maximum for all but the most disturbed clusters. We show the mass function in the box at various redshifts in Fig. 1.

\subsection{Adding galaxies}

We added galaxies to the simulation using a variant of the "halo model" for large-scale structure wherein gravitational clustering is described in terms of dark matter halos which form a biased tracer of the large-scale density field. Galaxies are distributed in halos following the dark matter profile with an occupation number which characterizes the efficiency of galaxy formation. This method produces galaxy distributions which are in agreement with those produced by semi-analytic models of galaxy formation (Kauffman et al. 1999; Somerville \& Primack 1999; Benson et al. 2000) and high resolution hydrodynamic simulations including star formation and feedback (Katz, Hernquist \& Weinberg 1999; Gardner et al. 2001; Pearce et al. 1999; White, Hernquist \& Springel 2001) and can match the observed low-order clustering statistics of galaxies (e.g. Jing et al. 1998; Benson et al. 2000; Seljak 2000a; Peacock \& Smith 2000; Scoccimarro et al. 2001; Scoccimarro \& Sheth 2001).

Our methodology is somewhat simpler than the full semi-analytic treatments described above, more closely approximating that of Kauffmann, Nusser \& Steinmetz (1997). For every output of the simulation we produce a halo catalogue by running a "friends-of-friends" (FoF) group finder with a linking length $b=0.2$. This procedure partitions particles into equivalence classes by linking together all particles separated by less than distance $b$. We keep all groups above 8 particles, which imposes a minimum halo mass of $3 \times 10^{11} h^{-1} M_{\odot}$. A slightly smaller minimum mass would be preferable, but with fixed dynamic range would come at the expense of less volume. For simplicity we take the halo "mass" to be the sum of the particles masses in the FoF group. We populate each halo with an integer number, $N$, of "galaxies". Each halo is a host to galaxies of two types. The first, or central galaxy, is placed at the center of mass and inherits the center of mass velocity. Any additional galaxies are assumed to be satellites

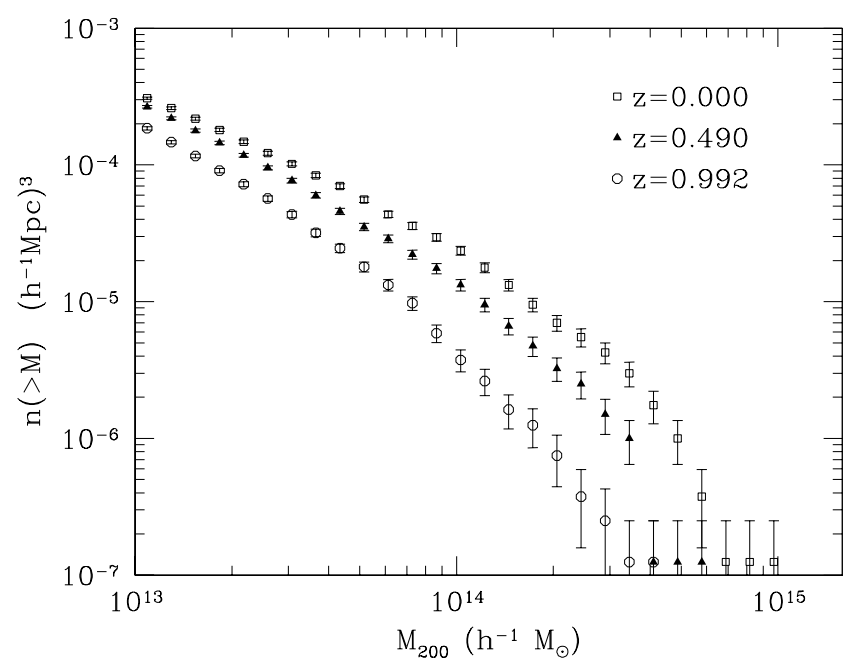

FIG. 1.- The 3D mass function of halos in our simulation box at $z=0,0.49$ and 0.99 . Masses are $M_{200}$, the mass enclosed within a radius, $r_{200}$, within which the mean density is 200 times the critical density at that redshift. Error bars indicate purely Poisson errors. 
and are laid down tracing the distribution of mass in the halo, including asymmetry and sub-structure, and inherit the velocity of the nearest dark matter particle. This spatial behavior is as seen in a recent hydrodynamic model of galaxy formation (White, Hernquist \& Springel 2001) and is assumed in the halo model. By having the galaxies trace the 3D density structure in the halo rather than an azimuthally averaged radial profile (such as the Navarro, Frenk \& White 1996 profile) we avoid producing artificially "spherical" clusters. For ease of later identification, we tag each galaxy with the mass of its parent halo and mark "central" galaxies as such.

The number of galaxies in each halo is drawn from a distribution whose moments we take from the semi-analytic models of galaxy formation of Kauffman et al. (1999) as fit by Sheth \& Diaferio (2001). Following Scoccimarro et al. (2001) we model the distribution of $N$ as a binomial. For simplicity we use the same $N(M)$ at all redshifts.

Unfortunately, a simple implementation of these algorithms poorly reproduce real observations because they under predict the observed numbers of galaxies by approximately a factor of three. The missing galaxies arise because the available models for the halo multiplicity function are calibrated to match particular flux-limited samples (e.g. the APM survey) rather than providing general expressions as a function of galaxy luminosity.

Since our ability to characterize the search for clusters depends critically on the actual numbers of galaxies as well as their spatial distribution, we adjusted the models to better match the observed density of galaxies. The basic problem is that the number of galaxies should vary with the minimum luminosity as $\Gamma\left[1+\alpha, L / L_{*}\right]$ where $\alpha$ is the faint-end slope of the luminosity function, modeled as a Schechter function (see Eq. 2) with characteristic luminosity $L_{*}$. The standard halo multiplicity expressions were normalized at a luminosity limit $L / L_{*} \sim 1 / 2$

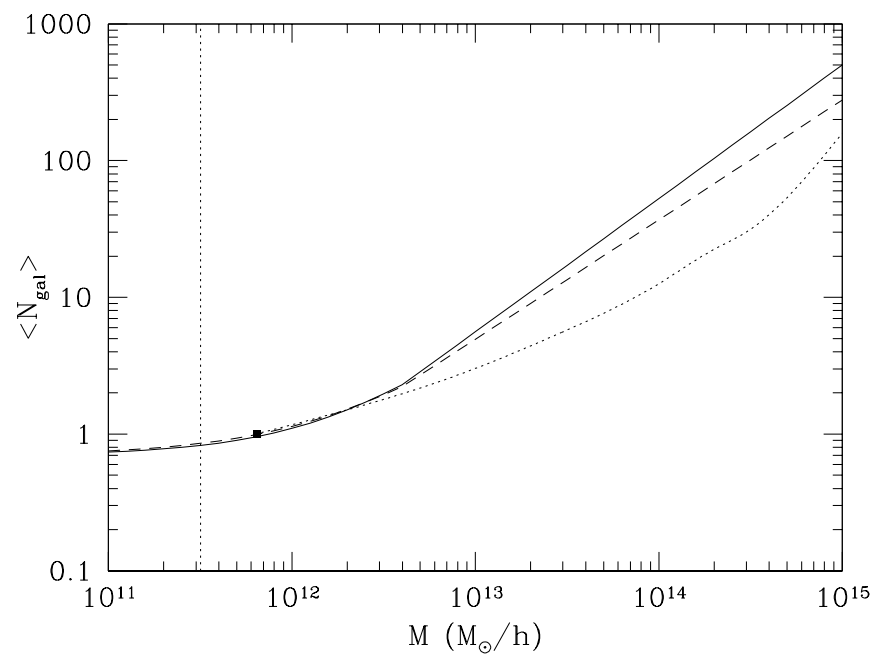

FIG. 2.- The mean number of galaxies in a halo of mass $M$. The dashed line shows the fit to the semi-analytic model of Kauffman et al. (1999), the dotted line the estimate of Peacock \& Smith (2000) which is zero below $10^{11.8} h^{-1} M_{\odot}$. The solid line is the functional form used in this work. The vertical dotted line marks the mass of an 8 particle halo. and our model surveys need to include galaxies down to $L / L_{*} \sim 1 / 10$ or even lower to correctly account for the observed number of galaxies. We achieve this by steepening the high-mass slope of the multiplicity function and including galaxies corresponding to lower mass halos. In clusters we roughly double the number of galaxies in a $10^{15} h^{-1} M_{\odot}$ cluster, and with a comparable increase in the number of galaxies in low mass halos we preserve the contrast between the clusters and the background. Because of the limited dynamic range in the simulations we cannot directly probe smaller mass halos, so we considered as 'galaxies' a fraction of the un-grouped particles in the simulation chosen so as to have about as many ungrouped galaxies as grouped galaxies. The ungrouped particles have similar clustering properties to the lowest mass halos.

With these modifications our galaxy sample maintains the properties of the spatial distribution needed for a realistic model while raising the comoving density of galaxies closer to the observed density. For example, the galaxy sample has an approximately power-law correlation function and power spectrum on small scales, over the range $0.5 h^{-1} \mathrm{Mpc}<r<10 h^{-1} \mathrm{Mpc}$ the galaxy correlation function is well fit by $\xi(r)=\left(r / r_{0}\right)^{-\gamma}$ with $r_{0}=5 h^{-1} \mathrm{Mpc}$ and $\gamma=1.8$ and $\sigma_{8}^{\text {gal }} \simeq 0.9$. With $\sim 450,000$ galaxies in the $200 h^{-1} \mathrm{Mpc}$ box at $z \simeq 0$, the total comoving density of galaxies is close to that implied by the LCRS (Lin et al. 1996) luminosity function.

\subsection{Simulating a field}

We simulate an observed field by "stacking" different slices through the box at earlier and earlier output times. We divide every output up into 6 "halves" (top, bottom, left, right, front, back) of $200 \times 200 \times 100 h^{-1} \mathrm{Mpc}$. A given observational field is then simulated by dividing the lineof-sight up into $100 h^{-1} \mathrm{Mpc}$ pieces stepping back from the observer. For each piece we choose one half of the box at the appropriate redshift, shifted perpendicular to the lineof-sight by a random amount using the periodicity of the simulation volume. A fraction of the galaxies in that half of the box are projected onto the sky at the appropriate location with the appropriate redshift, including the peculiar velocity of the galaxy. We have chosen $100 h^{-1} \mathrm{Mpc}$ as our sampling interval because it is large enough that edge effects are minimal even for rich clusters while being fine enough that line-of-sight integrals are well approximated by sums over the (static) outputs. However, even though only a small fraction of clusters lie within $r_{200}$ of a slice boundary, we decided to require that the orientation and offset change only on every second slice. Thus if we choose at one redshift the front of the box the next slice is required to the back. In this manner a cluster on the boundary is almost always included, though the periodicity of the box is artificial.

In addition to these fields we also generated "Poisson" fields in which the galaxy positions in each simulation box were randomized before being placed into the map. These fields were used to estimate likelihood thresholds for the cluster finding described in $\S$ 国. In practice, we found that the Poisson fields had such low likelihoods for clusters compared to the real data that they were of little use.

Although we assign galaxies to halos based on the mass 


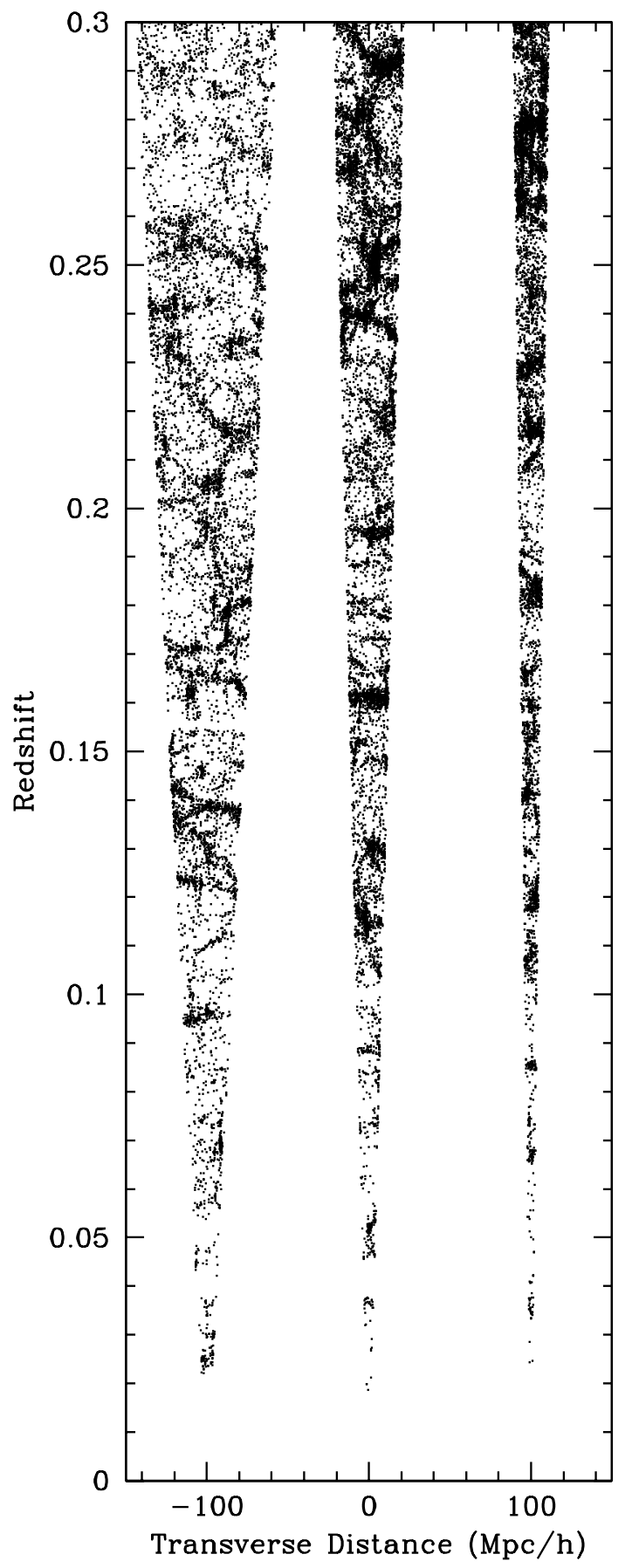

FIG. 3.- Wedge diagrams of parts of 3 of our fields. In each case we plot redshift against projected separation (comoving) in right ascension in a wedge $1.5^{\circ}$ thick. The surveys, offset for clarity, are our model MMT survey (left) subtending $6^{\circ}$ in RA; our model SDSS survey (middle) subtending $3^{\circ}$ in RA; and our model LSST survey (right) subtending $1.5^{\circ}$. Each dot represents a 'galaxy' and all galaxies are plotted. of their parent halo, we assign luminosities to the galaxies randomly based on a model luminosity function. The luminosity function enters our calculation only by defining the distance-dependent probability that a galaxy is sufficiently luminous to be included in the final catalog. We assume a luminosity function $\phi(L, z)$ where the redshift dependence enters only through the evolution of a characteristic luminosity $L_{*}(z)$ following a conservative $z_{f}=2$ burst evolution model. If at redshift $z$ we can detect galaxies brighter than $L(z)$ given our model flux limit, then the probability of including a galaxy at redshift $z$ is

$$
p(z)=\left[\int_{L(z)}^{\infty} \phi(L, z) d L\right]\left[\int_{0}^{\infty} \phi(L, z=0) d L\right]^{-1} .
$$

For a Schechter luminosity function,

$$
\phi(L)=\left(n_{*} / L_{*}\right)\left(L / L_{*}\right)^{\alpha} \exp \left(-L / L_{*}\right)
$$

the function becomes

$$
p(z)=\frac{\Gamma\left[1+\alpha, L(z) / L_{*}(z)\right]}{\Gamma[1+\alpha, 0]}
$$

where we will base our luminosity function on the LCRS Rband luminosity function (Lin et al.1996) with $\alpha=-0.70$. The resulting number of galaxies is very sensitive to the treatment of the low mass, low luminosity galaxies. Because our simulations do not treat low mass halos well, we modified the LCRS luminosity function so as to produce surveys with galaxy surface densities closer to those observed. For luminosities $L_{\text {cut }}<0.1 L_{*}$ we truncated the luminosity function as $\phi(L)=\left(L / L_{\text {cut }}\right) \phi_{L C R S}\left(L_{\text {cut }}\right)$. In a survey to $\mathrm{R}=20 \mathrm{mag}$, this modification increases the surface density of galaxies from 1100 per square degree to 1700 per square degree with no significant changes in the redshift distribution. Figure 4 shows the selection function $p(z)$ for a range of limiting magnitudes.

For simplicity we do not attempt to assign luminosities or colors to the galaxies, but characterize them only by the Gaussian uncertainty in their redshifts. For typical luminosity functions, the flux of a galaxy is sufficient to determine the redshift with an uncertainty of $\sigma_{z}=z / 2$. This sets an upper bound on the redshift uncertainties for nearby galaxies. For photometric redshifts we will explore $\sigma_{z}=0.05$ and 0.10 . For spectroscopic redshifts we used a very conservative uncertainty of $\sigma_{z}=0.01$ to smooth the distribution on scales somewhat larger than the velocity dispersions of rich clusters.

We considered three survey models motivated by the ongoing or proposed photometric and redshift surveys. The first example is a complete redshift survey to $R=20 \mathrm{mag}$ as might be conducted with the Hectospec fiber instrument on the $6.5 \mathrm{~m}$ MMT. This sample would be ten times deeper (in flux) than the current generation of redshift surveys (LCRS, $2 \mathrm{dF}$ and SDSS). The second example is motivated by the SDSS survey. It consists of a complete redshift survey to $\mathrm{R}=17.5 \mathrm{mag}$, a sparse, red galaxy-biased redshift survey to $\mathrm{R}=20 \mathrm{mag}$, and a photometric survey to $\mathrm{R}=22 \mathrm{mag}$. The Kauffman et al. (1999) model provides separate halo mass-dependent estimates for the number of red and blue galaxies. All galaxies brighter than $\mathrm{R}=17.5 \mathrm{mag}$ and $4 \%$ of the red galaxies ( $1 \%$ of all galaxies) between $R=17.5 \mathrm{mag}$ and $R=20 \mathrm{mag}$ are assigned 
spectroscopic redshifts while the remainder are assigned photometric redshifts. The remaining galaxies between $\mathrm{R}=17.5 \mathrm{mag}$ and $\mathrm{R}=20 \mathrm{mag}$ and the galaxies between $\mathrm{R}=20 \mathrm{mag}$ and $\mathrm{R}=22 \mathrm{mag}$ are assigned photometric redshifts. The final example is a deep photometric survey to $\mathrm{R}=24 \mathrm{mag}$ as might be done with the LSST. We assume the survey is conducted in an SDSS region and includes the SDSS spectroscopic redshifts. The properties of the model surveys are summarized in Table 1.

\subsection{Limitations}

The primary limitation in interpreting our results is that our model surveys consistently contain too few galaxies. For limiting magnitudes of $\mathrm{R}_{c}=20,22$ and 24 mag we have 1700,8700 and 32000 galaxies per square degree compared to observed counts of 2400, 14000 and 81000 galaxies per square degree based on the Gunn-r counts from McLeod \& Rieke (1995) and a color of $\mathrm{R}_{c}=r-0.35$. These undercounts are present despite our modifications to the halo multiplicity function and the luminosity function. In the absence of numerical resolution effects, simply scaling up $N(M)$ would not affect the clustering of our galaxies, but may not be the most physically realistic solution since it implies relatively low mass halos would be hosts to several galaxies. While it is plausible that the simulations undercount the halos which will be low luminosity galaxies or that the luminosity function genuinely turns down at low luminosity, it would not be physically realistic to raise our break luminosity above $L_{\text {cut }}=L_{*} / 10$.

However, if our model galaxy distribution adequately reproduces the statistics of real galaxy distributions, as seems to be the case, the primary consequence of the lower number of galaxies is to add Poissonian noise to our search. The Poisson noise level will be $20 \%, 27 \%$ and $60 \%$ higher than in a real survey to $R_{c}=20,22$ and 24 mag which is not a severe increase. Since real samples should have more galaxies, our results should be conservative.

A secondary limitation of our modeling is that we have treated the effects of evolution in the luminosity function very simply, using a passive evolution model in which stars form in a single burst at $z_{f}=2$. Particularly for the LSST field, where the median redshift is $z=0.6$, such a model is too simplistic. A more realistic model would require the identification and treatment of individual galaxy types. The inclusion of galaxy types whose evolution is faster than a passive model would help reduce the discrepancy in the number of galaxies. However, given the problems with the halo multiplicity function and the form of the luminosity function, we felt that adding a more detailed treatment of evolution should await a better underlying simulation.

\section{FINDING CLUSTERS}

Our objective is to automatically produce catalogs of cluster candidates from the synthetic fields which we can then check using our knowledge of the true mass distributions. We do this using the matched filter method described in $\$ 4.1$, adding some comments on how it can be adapted to real data or further improved in $\$ 4.2$. In $\$ 4.3$ we discuss the diagnostics we use to compare the output cluster catalog to the true clusters.

\subsection{The Matched Filter Algorithm}

We searched for clusters using an automated version of the Adaptive Mesh Filter (AMF) algorithm (Kepner et al. 1999), which is itself based on the "matched filter" algorithm of Postman et al. (1996).

We model the density of galaxies as a redshift-dependent background $\rho_{b}(z)$ and a distribution of $k=1 \cdots n_{c}$ clusters. Cluster $k$ is described by its angular position $\vec{\theta}_{k}$, (proper) scale length, $r_{c k}$, redshift $z_{k}$ and galaxy number $N_{k}$. At the corresponding angular diameter distance $D_{A}\left(z_{k}\right)$ the density of galaxies associated with the cluster is

$$
N_{k} \Sigma\left[\left(\vec{\theta}-\vec{\theta}_{k}\right) D_{A}\left(z_{k}\right) / r_{c k}\right] \delta\left(z-z_{k}\right)
$$

where we use a projected profile

$$
\Sigma(x) \propto \frac{1}{(1+x)^{2}}
$$

normalized by $\int_{0}^{c r_{c}} \Sigma(x) d^{2} \vec{\theta} \equiv 1$ for the angular distribution and (assuming redshift errors large compared to cluster velocity dispersions) a delta function for the redshift distribution. The simple, analytic profile defined by Eq. (5) provides a good match to a projected NFW (Navarro, Frenk \& White 1996) profile. We fixed the halo concentration to $c=4$ and the break radius to $r_{c}=200 h^{-1} \mathrm{kpc}$, as typical parameters for cluster-mass halos (e.g. Bullock et al. 2001). For a galaxy $i$ located at $\vec{\theta}_{i}$ and redshift $z_{i}$ with assumed Gaussian uncertainties characterized by $\sigma_{i}$ the expected density for cluster $k$ is

$$
\begin{aligned}
\rho_{k}\left(\vec{\theta}_{i}, z_{i}\right)=N_{k} \Sigma & {\left[\left(\vec{\theta}_{i}-\vec{\theta}_{k}\right) D_{A}\left(z_{k}\right) / r_{c k}\right] } \\
& \times \exp \left[-\left(z_{i}-z_{k}\right)^{2} / 2 \sigma_{i}^{2}\right] / \sqrt{2 \pi} \sigma_{i}
\end{aligned}
$$

and the predicted density for galaxy $i$ becomes

$$
\rho_{i}\left(\vec{\theta}_{i}, z_{i}\right)=\rho_{b}\left(z_{i}\right)+\sum_{k=1}^{n_{c}} \rho_{k}\left(\vec{\theta}_{i}, z_{i}\right)
$$

where the background model must also be modified to include the effects of the redshift uncertainties.

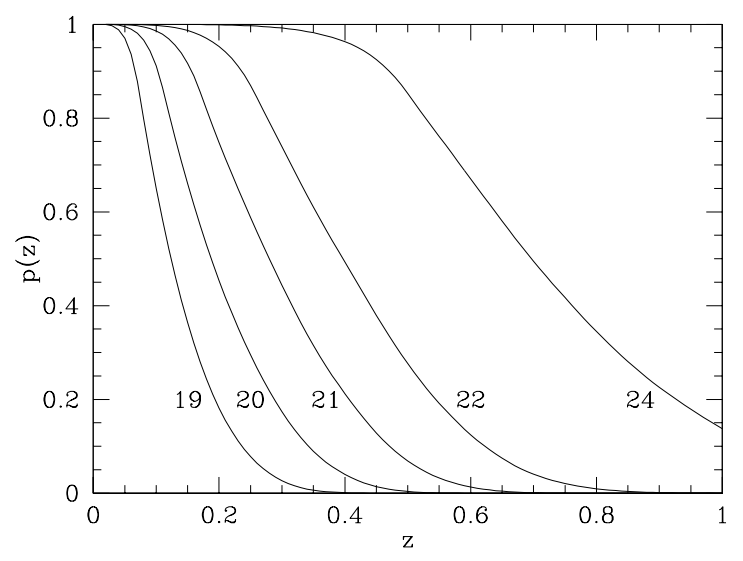

FIG. 4.- The "selection" functions adopted for surveys to the limiting $R$ magnitudes listed. The lines give the probability $p(z)$ that a galaxy at a given redshift is included in the final survey. 
The Gaussian redshift uncertainties model any information used to estimate the redshift of the galaxies in the survey. At its crudest this estimate comes only from the flux (magnitude) of the galaxy, and at its best it comes from direct spectroscopic redshifts. We are interested in the intermediate case where we possess photometric redshift estimates, presumably derived from galaxy colors, with accuracies in a range from $0.05 \lesssim \sigma_{z} \lesssim 0.1$.

The likelihood of the model over an area $A$ encompassing all the clusters and galaxies $i=1 \cdots n_{g}$ is

$$
\begin{aligned}
\ln \mathcal{L}= & -A \int d z \rho_{b}(z)-\sum_{k=1}^{n_{c}} N_{k} \\
& +\sum_{i=1}^{n_{g}} \ln \left[\rho_{b}\left(z_{i}\right)+\sum_{k=1}^{n_{c}} \rho_{k}\left(\vec{\theta}_{i}, z_{i}\right)\right] .
\end{aligned}
$$

which is derived from the Poisson statistics of galaxies distributed over infinitesimal bins in redshift and angle (this is termed the "fine" likelihood by Kepner et al. 1999, as compared to a "coarse" likelihood based on Gaussian statistics).

We build the model iteratively starting from a smooth background $\left(n_{c}=0\right)$. At each step we use the galaxy positions and redshifts as trial cluster centers, optimizing the likelihood with respect to the next cluster richness $N_{k}$ with $k=n_{c}$ but holding the properties of the background and all previous clusters fixed. We add the trial cluster producing the largest increase in the likelihood to the global model and then search for the next cluster, continuing the process until the likelihood gain drops below a threshold.

Our approach differs from that described by Kepner et al. (1999) in several respects. First, we make no use of the "coarse" (Gaussian) statistical model. After careful arrangement of the calculation and construction of linked lists, our execution time was not dominated by the optimization of the Poissonian likelihood with respect to $N_{k}$. Second, our density model explicitly includes the distribution and structure of previously found clusters. This automates the algorithm and provides a reasonable approach to separating overlapping clusters and minimizing multiple discoveries of the same cluster. In essence, we have combined the AMF algorithm for finding clusters with the Clean algorithm of radio astronomy for producing maps. Third, rather than simply clipping the redshift catalog to bracket the redshift of a trial cluster, we explicitly include the error-convolved redshift distribution of the cluster galaxies as part of the density model.

\subsection{Performance, Tuning $\&$ Refinements}

$\begin{array}{ccccc}\text { Name } & N_{\text {field }} & \text { Size } & n_{\text {gal }} & z_{50} / z_{75} / z_{90} \\ \text { MMTS } & 9 & 6.0^{\circ} \times 6.0^{\circ} & 1700 & 0.23 / 0.30 / 0.36 \\ \text { SDSS } & 9 & 3.0^{\circ} \times 3.0^{\circ} & 8700 & 0.38 / 0.48 / 0.57 \\ \text { LSST } & 4 & 1.5^{\circ} \times 1.5^{\circ} & 32600 & 0.59 / 0.77 / 0.95\end{array}$

\section{TABle 1}

Characteristics of the simulated fields. Number simulated, size of field, number density of galaxies (per square degree) and the redshifts encompassing $50 \%, 75 \%$ and $90 \%$ of the survey galaxies.
For theoretical convenience we defined our algorithm purely in terms of redshift uncertainties, although it would be trivial to redefine it in terms of luminosities and colors. A combination of the luminosity function and spectrophotometric models would provide predictions $m_{\text {est }}^{j}(z)$ for the measured magnitudes $m_{i}^{j}$ of galaxy $i$ in filters $j=1 \cdots n_{f}$ as a function of redshift and our Gaussian redshift error is replaced by the fit statistic between the model and the data. This could include a range of galaxy types and differences in galaxy properties between the field and clusters.

We ran our experiments with a fixed cluster scale $r_{c}=$ $200 h^{-1} \mathrm{kpc}$ and concentration $c=4$, although in theoretical models clusters have a range of scales, $100-500 h^{-1} \mathrm{kpc}$, and concentrations, $c \simeq 4-8$. We experimented with varying the scale radius and found that that the algorithm was biased towards allowing $r_{c}$ to become unreasonably large for some, but not all, cluster candidates. Although we did not conduct further experiments, the problem could be solved by adding a prior probability term for either the scale radius or the cluster mass to bias the solutions against finding overly large or massive clusters. The most natural prior is a simple model for the cluster mass function such as a $P(N) \propto 1 / N^{2}$ power-law. We also found that if we increased the outer fit radius too much (i.e. larger $c$ at fixed $r_{c}$ ) the algorithm systematically merged neighboring clusters. With $c=4$ this rarely happened, although occasionally a rich, nearby cluster was split into more than one 'candidate'.

We constructed our background model field-by-field based on a coarsely binned redshift distribution of the galaxies with their assigned redshifts (i.e. including the redshift error and scatter). The continuous distribution was then obtained by linear interpolation between the bins, which proved sufficient for our purposes and more stable than spline interpolation.

The initial distribution of likelihoods $\ln \mathcal{L}$ is relatively well modeled as a log-normal distribution with a tail to higher likelihoods. To set the termination point of our algorithm we fit the initial likelihood distribution to determine the mean and dispersion after rejecting likelihoods more than two standard deviations from the mean. We empirically set the stopping point at a likelihood threshold corresponding to the mean plus 1.3 standard deviations, which would mean that $90 \%$ of the galaxies were below the threshold for a Gaussian distribution.

Finally, we optimized only the properties of the new cluster in estimating the likelihood. The performance of the algorithm might be enhanced by simultaneously optimizing the richnesses of any overlapping clusters.

\subsection{Diagnostics}

After deriving the output cluster catalog we match it to both the input cluster catalog and the galaxy catalog. For each galaxy we have the probability $\rho_{b}$ that the galaxy is in the field and the probabilities $\rho_{k}$ that it is in any of the $k=1 \cdots n_{c}$ cluster candidates. We assigned galaxies to clusters by first finding the most probable cluster for the galaxy (the index $k$ with the maximum $\rho_{k}$ for the galaxy) and then assigning it to the cluster if $\rho_{k}>\rho_{b}$. For comparison to the fitted cluster number $N_{k}=N_{\text {fit }}$, we also counted the number of galaxies, $N_{\Delta}$, above a range of contrast thresholds where $\left(\rho_{k}>\Delta \rho_{b}\right)$. Our basic as- 
signment procedure used a contrast $\Delta=1$ and will have more background contamination than a higher threshold. We find that $N_{1}$ tracks $N_{\text {fit }}$ closely, but with more scatter. These estimates for the number of member galaxies can be compared to the true number, $N_{\text {true }}$, of galaxies assigned to cluster.

To match the output cluster catalogue to the input catalogue we used position and redshift information and in addition the modal parent halo mass of the galaxies assigned to the output cluster. This results in a unique match except in cases where a nearby rich cluster is broken into several candidates by the group finder, in which case that cluster can be flagged more than once. The matching is done in two directions, the best match from the input catalog to each cluster in the output list and the best match from the output catalog to each cluster in the input list. It is the latter, with duplicates trimmed, that we use to estimate completeness.

It occasionally happens that two clusters overlap on the sky and lie within $2 \sigma$ of each other in the redshift direction. In these cases our algorithm often misses one of the clusters, assigning its galaxies to the overlapping cluster. For the MMT survey this occurred slightly more than once per field, for a total of 12 missed clusters in the 9 fields. As the redshift error increases this number also increases, quadrupling for $\sigma_{z}=0.05$.

\section{RESULTS}

We illustrate our results by examining our ability to produce a catalog of clusters with masses above $2 \times 10^{14} M_{\odot}$, as these are the clusters which provide the greatest constraints for cosmology. Our assumption is that the catalog is an intermediate step, with further optical, X-ray or SZ observations being used to clean and calibrate the sample. Thus we discuss only the identification of clusters and their members rather than the derivation of physical parameters. The selection of the catalog will represent a trade-off between completeness and contamination, with the contamination arising either from real, but lower mass, clusters or complete artifacts. We use our knowledge of the true cluster properties to design selection procedures for attaining this goal (see Appendix).

Fig. 5 shows the distribution of matched clusters in likelihood and redshift for the MMT redshift survey, marking the ones above our $2 \times 10^{14} M_{\odot}$ mass threshold. As expected, higher mass and lower redshift lead to higher likelihoods, but there is no sharp boundary between high and low mass clusters. However, there is clearly a redshiftdependent likelihood threshold which would keep the completeness (fraction of $M \geq 2 \times 10^{14} M_{\odot}$ clusters found) high, the contamination (fraction of $M<2 \times 10^{14} M_{\odot}$ clusters or false detections) low, and both roughly independent of redshift. If we simplify the likelihood calculation (Eq. 8) by assuming a top hat cluster density profile, then we can show that the leading terms in the likelihood depend only on the number of galaxies in the cluster, with $\Delta \ln \mathcal{L} \propto N_{\text {true }}$ to lowest order. 7 As shown in Fig. 6, the likelihood scales in this manner for the data as well.

Thus, although there is considerable scatter due to differences in the structure of the cluster, the distribution of

\footnotetext{
${ }^{1}$ For very large numbers of galaxies the scaling becomes $\Delta \ln \mathcal{L} \propto$ $N_{\text {true }} \ln N_{\text {true. }}$.
}

galaxies and the cluster environment, we adopt a likelihood threshold designed to track the number of galaxies expected in a cluster of fixed mass. For a Schechter luminosity function of slope $\alpha$ and an evolving characteristic luminosity $L_{*}(z)$, this corresponds to a likelihood threshold which decreases as $\Gamma\left[1+\alpha, L(z) / L_{*}(z)\right]$ with redshift, where $L(z)=4 \pi D_{\mathrm{lum}}^{2} F$ is the luminosity corresponding to the survey flux limit. For further experiments we set our survey thresholds using our knowledge of the true masses. In a real survey the thresholds would have to be calibrated using clusters of known mass. Fig. 5 illustrates the redshift-dependent likelihood cuts $\Delta \ln \mathcal{L}_{\text {cut }}(z)$ for a range of local normalizations $\Delta \ln \mathcal{L}_{\text {cut }}(z=0)$.

We used the most common parent halo mass of the galaxies identified with a cluster candidate to identify the input halo corresponding to the candidate. This procedure led to multiple identifications of the most probable, low redshift, massive clusters where we would find lower likelihood satellite clusters most of whose galaxies are members of the more massive cluster. This is at least in part due to our fixed filter profile whose $r_{c}=0.2 h^{-1} \mathrm{kpc}$ is somewhat smaller than the break radius of the most massive clusters. We have automatically filtered these out by dropping cluster candidates with the same modal mass as a more likely cluster and within a projected separation of $1 h^{-1} \mathrm{Mpc}$ and a redshift difference of $\Delta z=0.05$. For the MMTS model survey these represented $5 \%$ of the cluster candidates found. In a real survey, where we would lack the knowledge of the parent masses, these false candidates would be initially identified as lower mass clusters in the halo of a massive cluster and then eliminated by more careful modeling of the structure of the most massive candidates.

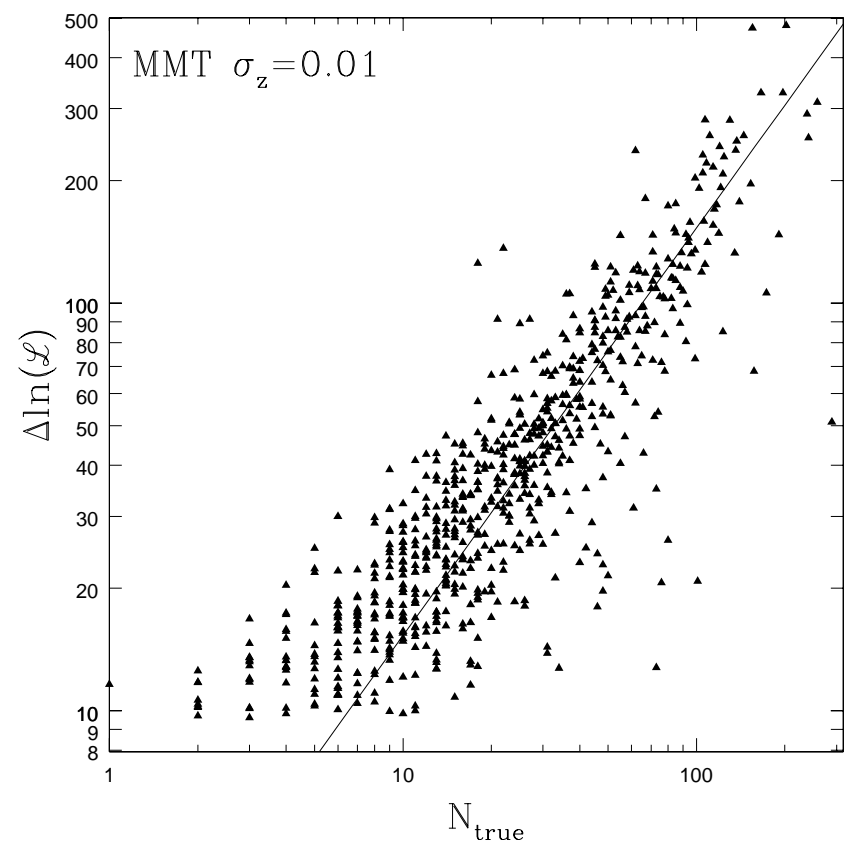

FIG. 6.- Likelihoods for $M \geq 2 \times 10^{14} M_{\odot}$ clusters as a function of their true galaxy number $N_{\text {true }}$. The line is a linear fit $\Delta \ln \mathcal{L} \propto N_{\text {true }}$ for the systems with $N_{\text {true }} \geq 10$. 


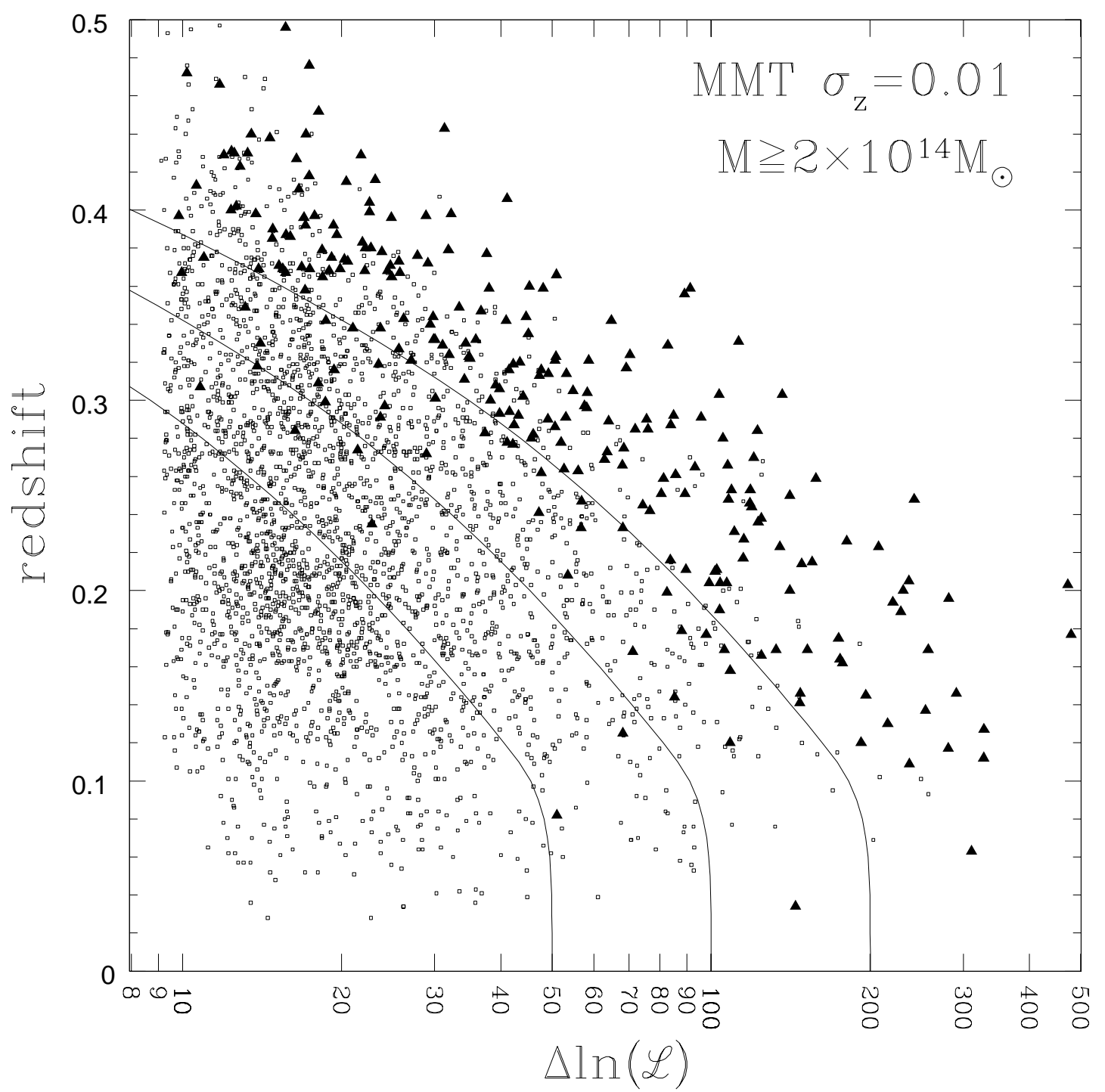

FIG. 5. - The distribution of candidate clusters in likelihood and redshift. Clusters with masses $M \geq 2 \times 10^{14} M_{\odot}$ are shown by the large, filled triangles, while those with masses $M<2 \times 10^{14} M_{\odot}$ are shown by small, open squares. The curves show cluster selection boundaries for zero redshift likelihoods of 50, 100 and 200 . 
We also find real high mass clusters with anomalously low likelihoods. Many of these are edge effects, where the cluster is within $0.5 h^{-1} \mathrm{Mpc}$ of the field edge. We made no modifications to our algorithm to adjust the likelihoods for the field edges. We also find a very small number of overlapping high mass clusters in which one cluster absorbs galaxies from the other leading to overly high likelihood cluster and one overly low likelihood cluster. While the low likelihood cluster may drop below our selection thresholds, because it overlaps with a cluster above the threshold, later studies with more accurate redshifts will correct the confusion. Errors in finding the clusters in the original N-body simulation can also be interpreted as completeness and contamination problems - the FoF algorithm can combine merging clusters into a single more massive cluster which our search algorithm then rediscovers as a pair of merging clusters of lower likelihood than expected given the FoF mass estimate. This effect is somewhat exacerbated by our use of the canonical but relatively large linking length $b=0.2$.

The next step in defining a sample is to select $\Delta \ln \mathcal{L}(0)$, the zero redshift normalization of the likelihood selection function. Fig. Ifillustrates how the completeness and false positive fraction depend on the likelihood threshold for each of our model surveys. We include all cluster candidates inside the redshift encompassing $90 \%$ of the survey galaxies (see Table 1). The equivalent curves for lower redshift thresholds will have lower false costive rates for the same completeness because the number of false positives rises with redshift. We define the completeness as the fraction of clusters inside this redshift limit with masses above $M_{200} \geq 2 \times 10^{14} h^{-1} M_{\odot}$ which are found in the cluster catalog with likelihoods above the threshold. As we raise the likelihood threshold, the completeness declines. False positives are candidate clusters with likelihoods exceeding the threshold which do not correspond to a $M_{200} \geq 2 \times 10^{14} h^{-1} M_{\odot}$ cluster. We distinguish two types of false positives - candidates identified with real but less massive groups, and candidates we could not identify with any group (a "false" group). Higher likelihood thresholds reduce the numbers of false positives.

Complete redshift surveys, as illustrated by the MMT survey in Fig. 7, easily produce very complete cluster samples to redshifts well past the survey median.2 While there are few false groups, the overall false positive fraction is significant and it is probably impossible to eliminate this problem. When we combine a noisy mass estimator (see below) with the very steep cluster mass function (see Fig. 1), many apparently massive clusters will be lower mass clusters with overestimated masses (a form of Malmquist bias). Most of the false positives in the MMT survey are real groups or clusters in the mass range $3 \times 10^{13} h^{-1} M_{\odot} \leq M_{200} \lesssim 10^{14} h^{-1} M_{\odot}$ (see Fig. 7). We discuss this mathematically in an Appendix. The completeness of the survey at low redshift is underestimated by our basic matching software. Of the 31 clusters missed for low likelihood thresholds, 3 (7) are within 1 (4) arcmin of a field edge and 4 have virial radii overlapping

\footnotetext{
${ }^{2}$ Bear in mind that our model for a redshift survey is very conservative, since a real redshift survey has velocity measurement errors under $100 \mathrm{~km} / \mathrm{s}$ and even rich clusters have velocity dispersions not much larger than $1000 \mathrm{~km} / \mathrm{s}$, while a $1 \%$ redshift error at $z=0.1$ corresponds to $3000 \mathrm{~km} / \mathrm{s}$.
}

that of another massive cluster with a redshift difference smaller than $2 \sigma_{z}$. As we change the mass threshold, we can maintain high completeness out to the redshift where the typical cluster at the mass threshold contains three galaxies, an effect which is well described by the Poisson model for the survey described in the Appendix.

As we switch from spectroscopic redshifts information to photometric redshifts the completeness achievable for a given false positive rate declines, as illustrated in Fig. 7 by the SDSS and LSST survey models. For runs with larger errors than shown here we even have difficulty performing the match between the input and output catalogs using the most common parent halo mass of the galaxies identified with a given cluster candidate.

Next we selected a likelihood cutoff where we estimate that the survey would be $80 \%$ complete and determined the completeness and false positive fractions of the resulting catalogs as a function of redshift (Fig. 8). Because of the design of the likelihood cut function, the completeness is nearly constant out to the redshift encompassing $90 \%$ of the survey galaxies. The false positive fraction generally rises with redshift, and catalogs extending to lower redshifts can have significantly lower contamination for the same level of completeness. The apparent drop in the completeness of the MMT model survey at low redshift is due in part to edge effects but also to the fact that we have required an extremely high likelihood threshold to remove low mass systems. The false positive rate rises faster with redshift in the SDSS survey because complete redshift information is available only for the nearby galaxies $(z<0.2)$. At these low redshifts, the addition of the deeper photometric catalog to the redshift data appears to improve the performance significantly.

The redshift dependence of the completeness is well defined by the Poisson model for the survey developed in the Appendix. For a likelihood threshold roughly corresponding to the number of galaxies in a cluster at the threshold mass, the survey will be nearly complete up to the redshift where the average number of galaxies in the threshold mass cluster drops below about 3 galaxies. The Poisson model works less well for explaining the fraction of false positives. Adding small number of background galaxies to each cluster does explain the rapid rise in the false positive fraction with redshift. The model underpredicts the false positive fraction at lower redshifts, probably because the contamination from the background distribution of galaxies is poorly described by a simple Poisson model.

Finally, we explore the correlation of our model cluster parameters with the true properties of the cluster. Fig. 9 compares estimates for the number of cluster galaxies with the true number. The number of galaxies estimated in the likelihood, $N_{c}$, closely matches the true number in rich clusters. But, in a catalog selected based on the cluster likelihood, we tend to find clusters with small galaxy excesses compared to the real cluster. Roughly speaking, the fit parameter $N_{c}$ usually finds $5-10$ more galaxies than were actually in the cluster. We can also estimate the number of galaxies by counting the number of galaxies $N_{\Delta}$ whose probability of being a cluster member exceeds their probability of being in the background by a factor of $\Delta$. The number at unit contrast, $N_{1}$, tracks $N_{\text {fit }}$ closely with some additional scatter. Higher contrast values provide better discrimination against background contamination. 


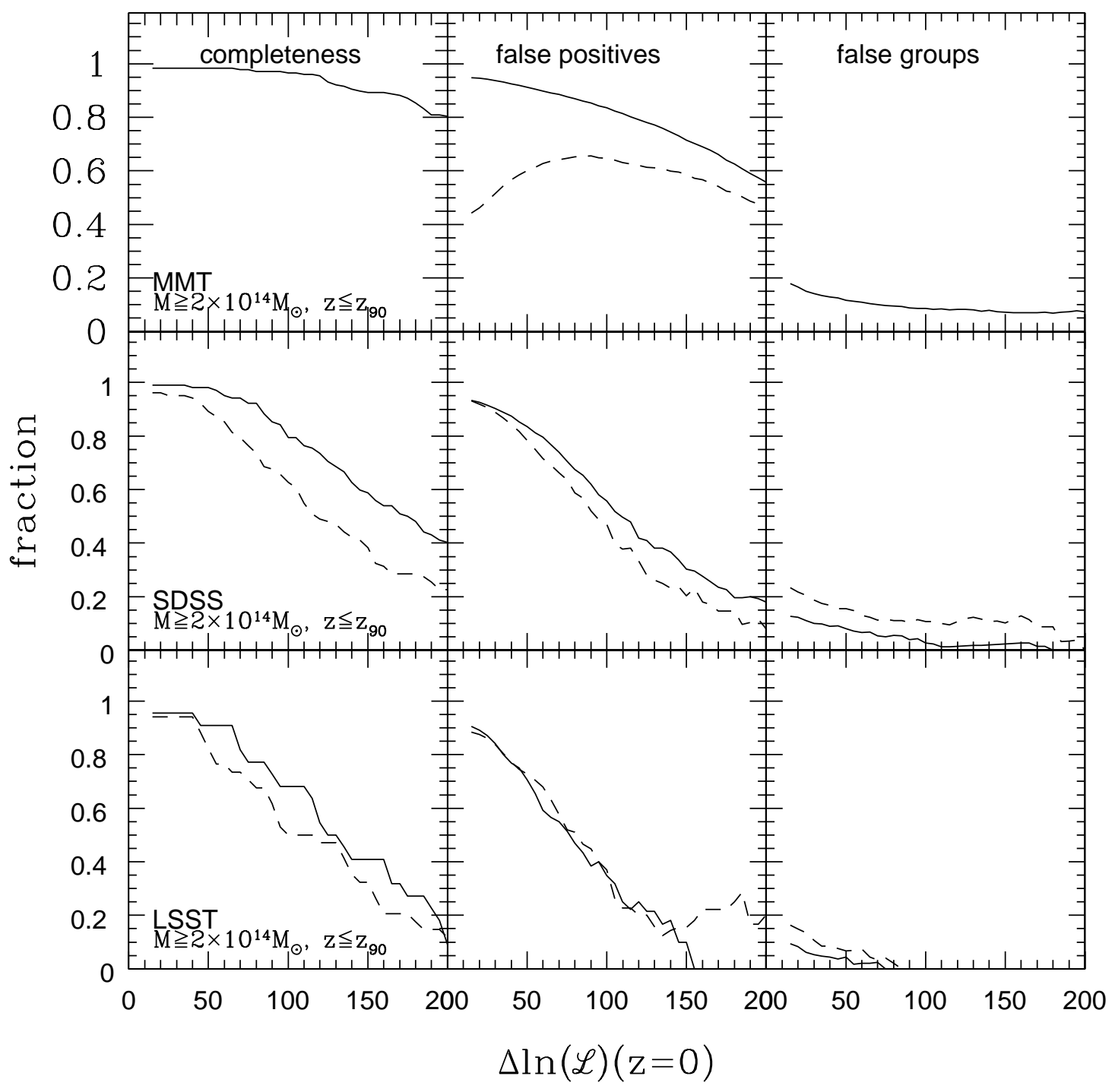

FIG. 7.- The survey completeness and false positive rates. The top, middle and bottom rows illustrate the properties of the MMTS, SDSS and LSST model surveys as a function of the zero redshift likelihood cut $\ln \mathcal{L}(z=0)$. The left column shows the completeness defined by the fraction of $M_{200} \geq 2 \times 10^{14} h^{-1} M_{\odot}$ clusters found in the survey out the redshift encompassing $90 \%$ of the survey galaxies. The middle column shows the false positive fraction defined by the fraction of cluster candidates above the likelihood threshold which are not $M_{200} \geq 2 \times 10^{14} h^{-1} M_{\odot}$ clusters. The right column shows the false group fraction defined by the fraction of cluster candidates above the likelihood threshold which are not identified with any input cluster. For the MMT survey the dashed line in the false positive column shows the fraction of candidates which correspond to slightly less massive clusters with $3 \times 10^{13} h^{-1} M_{\odot} \leq M_{200}<2 \times 10^{14} h^{-1} M_{\odot}$. For the SDSS and LSST surveys the line patterns show the assumed photometric redshift errors of $\sigma_{z}=0.05$ (solid) and 0.10 (dashed). 


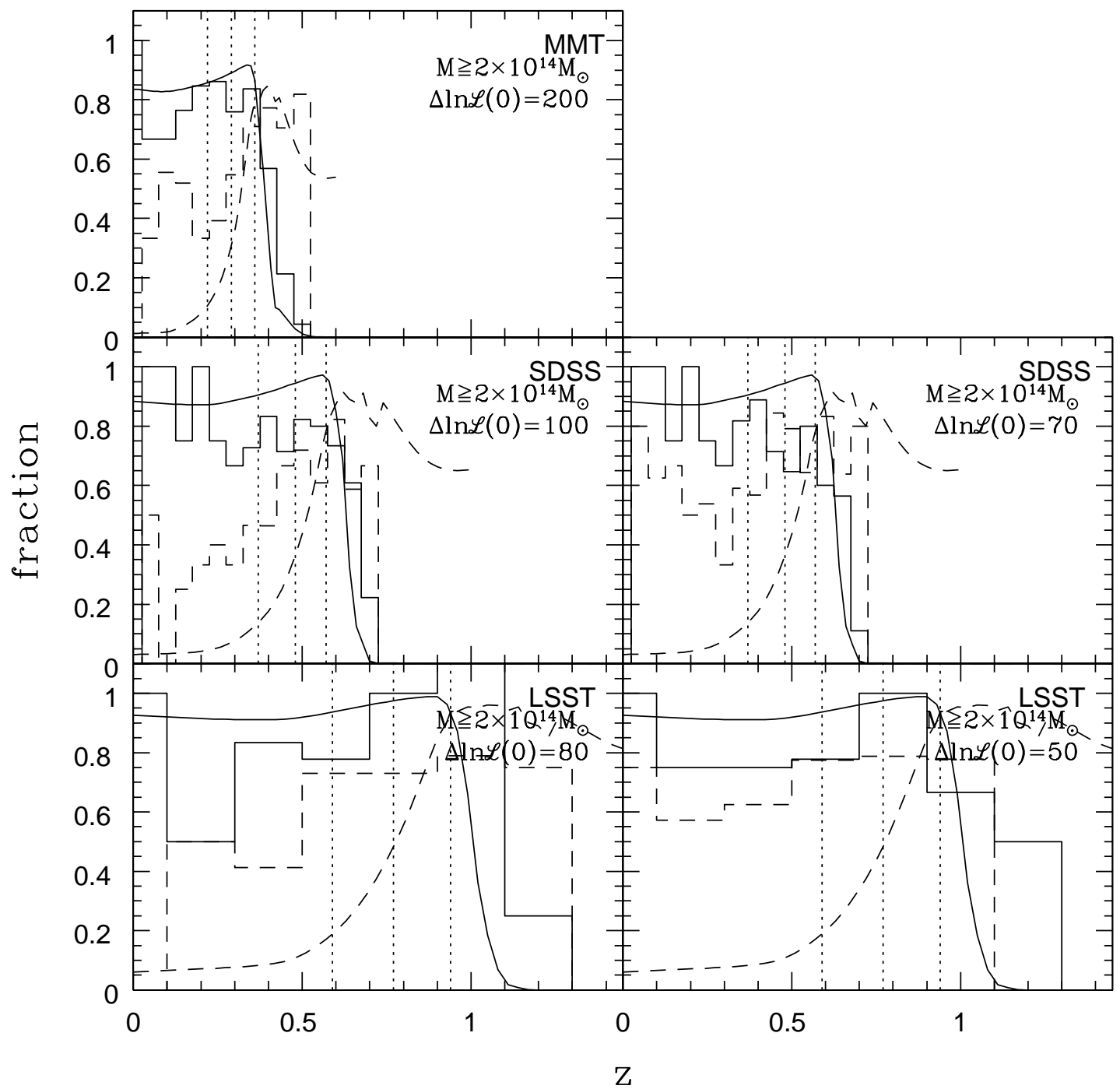

FIG. 8. - The survey completeness and false positive rates. The top, middle and bottom rows illustrate the properties of the MMTS, SDSS and LSST model surveys as a function of redshift. The columns show the effects of increasing errors in the redshift estimates. These are fixed to $\sigma_{z}=0.01$ for the MMT survey and are $\sigma_{z}=0.05$ (left) and 0.10 (right) for the SDSS and LSST model surveys. The solid histograms show the completeness, the fraction of $M_{200} \geq 2 \times 10^{14} h^{-1} M_{\odot}$ clusters found by the survey, and the dashed histogram show the fraction of false positives in the survey. This includes both real, but less massive clusters and false groups, but is generally dominated by real clusters with $3 \times 10^{13} h^{-1} M_{\odot} \leq M_{200}<2 \times 10^{14} h^{-1} M_{\odot}$. The solid (dashed) curves show the Poisson model for the completeness (false positive fraction) derived in the Appendix. The vertical dashed lines mark the redshift encompassing $50 \%, 75 \%$ and $90 \%$ of the survey galaxies. 
Fig. 9 also compares $N_{2}$ and $N_{4}$ with $N_{\text {true }}$. At an intermediate contrast $\Delta=2, N_{2}$ is less biased systems with small numbers of galaxies but begins to underestimate the numbers of galaxies in systems with large numbers of galaxies. These trends become clearer for the higher contrast level of $\Delta=4$. Inspection of individual systems with extreme ratios $N_{\text {fit }} / N_{\text {true }}$ provides no guidance towards an improved estimator. They tend to be relatively massive systems with modest numbers of galaxies where the finger-of-god from the velocity dispersion overlaps a larger than average number of galaxies.

Finally we can use the probability that any galaxy is a cluster member to estimate the cluster redshift. The estimates scale as expected, as shown in Fig. 10. In general it is possible to compute any cluster property (e.g. velocity dispersion) using such a probability weighting. We shall defer discussion of such estimators to a future publication.

\section{DISCUSSION}

Our tests of the matched filter method for finding clusters in optical surveys with either photometric or spectroscopic redshifts show that it is an efficient and reliable means of identifying massive clusters even when the redshift estimates are crude. In redshift surveys, where cluster surveys have usually used FoF methods rather than matched filters, the method works extremely well. By selecting clusters using a redshift-dependent likelihood threshold roughly tracking the expected number of galaxies from a cluster of fixed mass, we can construct catalogs with high completeness, low contamination and both varying little with redshift. The method automatically assigns a probability that each galaxy is a member of any cluster, which can be used in the estimate cluster properties such as redshift or velocity dispersion.

Both the completeness and the contamination in our

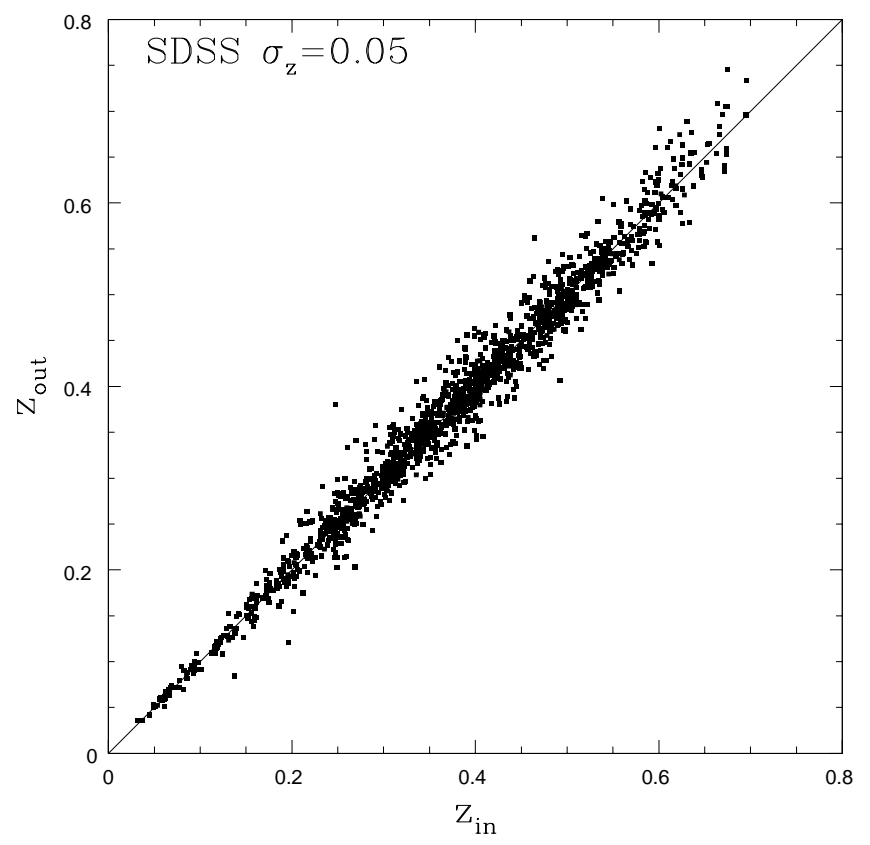

FIG. 10.- Estimated versus true cluster redshifts. mock surveys can be understood in part using a simple analytic model (described in the Appendix). The largest effect is the well known scatter in optical richness at a fixed cluster mass. Due to the steeply falling mass function of clusters this scatter implies that any sample selected on the basis of a fixed number of galaxies will be contaminated by abnormally rich, low-mass clusters. In our mock surveys this is by far the largest effect, with "false" clusters being almost entirely absent. We find that the false positive rate increases with redshift, so that our samples will have less contamination if restricted to a lower redshift cutoff than the 90th percentile we have assumed throughout. In any case, the likelihood threshold can be adjusted to modify either the desired completeness or contamination.

The addition of photometric data for fainter galaxies to a redshift survey, as in the SDSS at low redshift, considerably improves the detection of clusters over the redshift data alone. The redshift catalog, by pinning down the foreground contamination, probably improves the detection of higher redshift clusters which are detectable only in the photometric data.

We thus expect that the matched filter method can in future be used to construct large samples of clusters to "modest" redshifts, though follow-up observations will be necessary to clean the sample. Apart from clusters lying near the edge of our fields, the most common misidentification was to split very large clusters into a core and satellite population. This occurred due to our assumption of fixed core radius and concentration. The second most common misidentification was when two massive clusters overlapped, with one 'stealing' galaxies from another and causing it to drop below our likelihood threshold. In both cases any confusion would be immediately eliminated by more careful follow-up observations and modeling of the cluster region.

Our investigation is but a first step, and further work is needed. The primary limitation of our mock surveys is the inadequacy of current methods for populating dark matter halos with galaxies. Our simulations have too few galaxies compared to real surveys (by factors 30\%, $40 \%$ and $60 \%$ for $\mathrm{R}<20,22$ and $24 \mathrm{mag}$ ). While simulations with a higher dynamic range would be a step in the right direction, significant uncertainties remain in the modeling of $N(M)$ and how it depends on luminosity and redshift. Encouragingly our results should be conservative in this respect. It is also necessary to apply this method to real data, to uncover any failure modes which have been missed by the simulations.

\section{ACKNOWLEDGMENTS}

We would like to thank Jeremy Kepner for comments on the manuscript. C.S.K. was supported by the Smithsonian Institution. M.W. was supported by a Sloan Fellowship and the National Science Foundation. Simulations were carried out at CPAC, through grants PHY-9507695.

\section{APPENDIX}

\section{POISSON THEORY OF COMPLETENESS}

The likelihood of finding a cluster is largely controlled by the number of member galaxies. This allows us to make a model for the tradeoff between completeness and contam- 


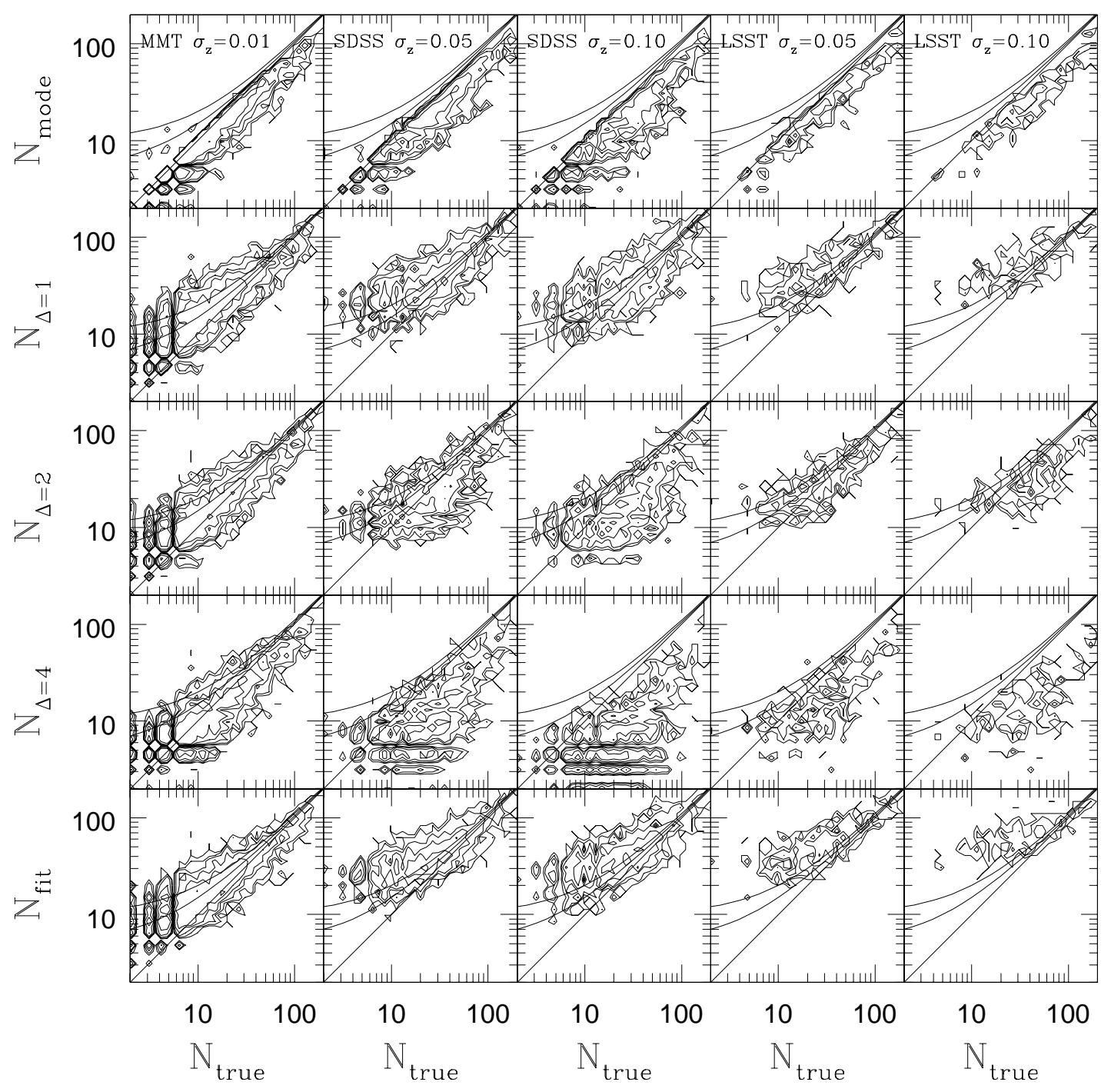

FIG. 9.- Density contours for various estimates of the number of galaxies in the cluster versus the true number of galaxies $N_{\text {true }}$ From bottom to top we compare the true number of galaxies to the estimated number from the likelihood function $N_{\text {fit }}=N_{c}$, the number of galaxies $N_{\Delta=4}, N_{\Delta=2}$ and $N_{\Delta=1}$ with a likelihood contrast relative to the background larger than a factor of $\Delta=4,2$ and 1 respectively, and the number of real cluster galaxies with a likelihood contrast relative to background larger than unity $N_{\text {mode }}$. The contours are spaced by factors of 2 in the density. The smooth curves show lines where $N=N_{\text {true }}, N=N_{\text {true }}+5$ and $N=N_{\text {true }}+10$. 
ination. If the expected number of galaxies in a cluster of mass $M$ and redshift $z$ is $\langle N\rangle=N_{0}(M) p(z)$ (see $\S 3.3$ ) and the halo mass function is $d n / d M$ then the number of clusters with $N_{\text {obs }}$ galaxies is

$\frac{d n}{d N_{\text {obs }}}=\int d V \int d M \frac{d n}{d M} \frac{\langle N(M, z)\rangle^{N_{\text {obs }}}}{N_{\text {obs }} !} \exp (-\langle N(M, z)\rangle)$

for volume element $d V$ and assuming Poisson statistics, expected for the high mass end of the mass function. If we search for clusters of mass $M \geq M_{0}$, the total number within redshift $z$ is

$$
N_{\text {tot }}\left(\geq M_{0}\right)=\int_{0}^{z} d V \int_{M_{0}}^{\infty} d M \frac{d n}{d M} .
$$

The cluster likelihood roughly scales as $\Delta \ln \mathcal{L} \propto N$ so we will find clusters above a fixed mass threshold if we scale the likelihood or the number of members with the expectation value. The threshold is set by the number of galaxies at $z=0, n_{0}$, and then decreases with redshift as $n(z)=n_{0} p(z)$. The cluster sample will contain

$N_{\text {fnd }}\left(\geq M_{0}, \geq n_{0}\right)=\int_{0}^{z} d V \int_{M_{0}}^{\infty} d M \frac{d n}{d M} P[n(z),\langle N(M, z)\rangle]$

galaxies above the mass threshold, where

$$
P[n, N] \simeq \frac{\Gamma[1+n, N]}{\Gamma[1+n]}
$$

is the fraction of clusters expected to have $N$ galaxies containing at least $n$ galaxies. The completeness of a sample selected with this criterion is $N_{\text {fnd }} / N_{\text {tot }}$. Since the likelihood depends only on the number of galaxies, we also find false positives from lower mass clusters with galaxy membership above the threshold. The number of false positives is

$N_{\text {false }}\left(<M_{0}, \geq n_{0}\right)=\int_{0}^{z} d V \int_{0}^{M_{0}} d M \frac{d n}{d M} P[n(z),\langle N(M, z)\rangle]$,

and the fraction of cluster candidates where are false positives is $N_{\text {false }} /\left(N_{\text {fnd }}+N_{\text {false }}\right)$.

We can extend this basic theory to a more realistic model for a cluster survey with two modifications. First, a cluster must contain a minimum number of galaxies, $N_{\text {thresh }} \simeq 3$, to be detected. This lower bound corresponds to the likelihood threshold of the catalog, below which the candidates are dominated by true false positives with no correspondence to any cluster. We implement it in the Poisson model by using a threshold $n(z)=\max \left(n_{0} p(z), N_{\text {thresh }}\right)$. Second, the cluster catalogs are also contaminated by unrelated galaxies. These chance projections alter the apparent number of galaxies associated with a cluster. Based on Fig. 9 we model these chance projections as a Poisson process with an expectation value of $N_{b} \simeq 5$. For a cluster expected to have $N_{c}$ galaxies and a detection threshold of $i$, the probability of the cluster including chance projections having at least $n$ galaxies at least $j$ of which are cluster members is

$$
P\left(n, j \mid N_{c}, N_{b}\right)=\sum_{i=0}^{n-j} P\left(n-i \mid N_{c}\right) \frac{N_{b}^{i}}{i !} \exp \left(-N_{b}\right) .
$$

We are taking the sum $i$ over the possible level of background contamination, weighted by its Poisson likelihood given the value of $N_{b}$, multiplied by the probability that the cluster will contain enough galaxies for the sum of the number in the cluster and in the background to reach the threshold.

We illustrate the behavior of this model in Fig. 8. We fixed $N_{\text {thresh }}=3$ and we adjusted $n(0)$ to produce a low redshift completeness slightly above the average observed completeness. We scaled it to be slightly above because we lose some clusters due to effects not in the model (edges, overlapping clusters). We fixed the amount of background contamination to $N_{b}=5,10$ and 15 for the MMT SDSS and LSST model surveys based very crudely on the offset between $N_{f i t}$ and $N_{t r u e}$ in Fig. 9. The Poisson model describes the completeness of the survey well, matching the extended region of nearly constant completeness followed by a sharp drop produced by the requirement for a finite number of galaxies $N_{\text {thresh }}$ in a cluster. The model describes the false positive fraction less well. The rapid rise in the false positive fraction near the drop in the completeness is due to the Poisson fluctuations in the contamination. However, the overall distribution of false positives cannot be explained by the Poisson model.

The limitation of the Poisson model is implicit in the wide range of likelihoods found for a fixed true number of galaxies (see Fig. 6). While the likelihood roughly scales with the true number of galaxies in the cluster, there is significant scatter about the general trend. Clusters differ not only in their total galaxy content, but also in their internal structure (break radius, concentration), the sampling of the internal structure, and the density of their local environment. Any effects which increase the scatter between the likelihood and $N_{\text {true }}$ will produce more false positives for a fixed level of completeness. The background contamination is also more complicated than a the simple Poisson model, since the background galaxies are themselves clustered. For example if the average background contamination is $N_{b}=4$ but galaxies are always clustered in pairs, the likelihood of 6 contaminating galaxies is nearly doubled.

\section{REFERENCES}

Abell G.O., 1958, ApJS, 3, 211

Benson A.J., et al., 2000, MNRAS, 311, 793

Blanchard A., Sadat R., Bartlett J.G., le Dour M., 2000, å, 362, 809

Bullock, J.S., Kolatt, T.S., Sigad, Y., Somerville, R.S., Kravtsov, A.V., Klypin, A.A., Primack, J.R., \& Dekel, A., 2001, MNRAS, 321,559

Carlstrom J.E., et al., 2000, Phys. Scripta, 85, 148

Dalcanton J., 1996, ApJ, 466, 92

Dalton G.B., Efstathiou G., Maddox S.J., Sutherland W.J., 1992, ApJ, 390, L1

Ebeling H., et al., 1998, MNRAS, 301, 881

Edge A., Stewart G.C., Fabian A.C., Arnaud K.A., 1990, MNRAS, 245,559

Gardner, J.P._. Nornquist, L., Weinberg, D.H., 2001, ApJ, 559, 131 astro-ph/9911343

Geller, M.J., \& Huchra, J.P., 1983, ApJS, 52, 61

Geller, M.J., 1994, RAS Canada, 88, 283

Gioia I.M., et al., 1990, ApJS, 72, 567

Haiman Z., Mohr J., Holder G., 2001, ApJ, 553, 545

Henry J.P., Arnaud K.A., 1991, ApJ, 372, 410

Henry J.P., 2000, ApJ, 534, 565

Hernquist, L. \& Katz, N., 1989, ApJS, 70, 419

Huchra, J.P., \& Geller, M.J., 1983, ApJ, 265, 356 
Jing Y.P., Mo H.J., Borner G., 1998, ApJ, 494, 1

Jones L.R., et al., 1998, ApJ, 495, 100

Kauffmann G., Colberg J.M., Diaferio A., White S.D.M., 1999, MNRAS, 303, 188

Kauffmann G., Nusser A., Steinmetz M., 1997, MNRAS, 286, 795

Katz N., Hernquist L., Weinberg D.H., 1999, ApJ, 523, 463

Kepner, J., Fan, X., Bahcall, N., Gunn, J., Lupton, R., \& Xu, G., 1999, ApJ, 517, 78

Lin, H., Kirshner, R.P., Shectman, S.A., Landy, S.D., Oemler, A., Tucker, D.L., \& Schechter, P.L., 1996, ApJ, 464, 60

Lumsden S.L., Nichol R.C., Collins C.A., Guzzo L., 1992, MNRAS, 258,1

McLeod, B.A., \& Rieke, M.J., 1995, ApJ, 454, 611

Navarro J., Frenk C.S., White S.D.M., 1996, ApJ, 462, 563

Ostriker J., Steinhart P.J., 1995, Nature, 377,600

Peacock J.A., 2000, MNRAS, 318, 1144 astro-ph/0002013

Peacock J.A., Dodds S.J., 1996, MNRAS, 28I Th

Peacock J.A., Smith R.E., 2000, preprint astro-ph/0005010

Pearce, F.R. et al., 1999, ApJ, 521, 99

Pierpaoli E., Scott D., White M., 2001, MNRAS, 325, 77

Postman M., et al., 1996, AJ, 111, 615

Press W.H., Schechter P., 1974, ApJ, 187, 452

Ramella, M., Diaferio, A., Geller, M.J., \& Huchra, J.P., 1994, AJ, 107,1623

Ramella, M., Pisani, A., \& Geller, M.J., 1997, AJ, 113, 483

Reblinsky K., Bartelmann M., 1999, A\&A, 345, 1

Romer A.K., et al., 2000, ApJS, 126, 209

Rosatti P., Della Ceca R., Burg R., Norman C., Giacconi R., 1995, ApJ, 445, L11

Scharf C.A., et al., 2000, ApJ, 528, L73

Scoccimarro R., Sheth R., 2001, preprint

Scoccimarro R., Sheth R., Hui L., \& Iain_B_ 2001_ApJ, 546, 20

Seljak U., 2000, MNRAS, 318, 203 [astro-ph/0001493]

Sheth R., Diaferio A., 2001, MNRA5, 322, yo1

Somerville R., Primack J., 1999, MNRAS, 310, 1087

van Haarlem M.P., Frenk C.S., White S.D.M., 1997, MNRAS, 287, 817

Vikhlinin A., et al., 1998, Ap.J, 503,77

White M., 2001, A\&A, 367, 27 astro-ph/0011495

White M., Hernquist L., Springel V., 2001, ApJ, 550, L129

White M., Springel V., Hernquist L., 2001, in preparation.

White R., et al., 1999, AJ, 118, 2014 